\title{
Optimum Design of Haptic Seat for Driving Simulator
}

\author{
Osama Halabi , Mariam Ba Hameish, Latefa Al-Naimi, Amna Al-Kaabi \\ Compute Science and Engineering Department \\ Qatar University \\ Doha, Qatar
}

\begin{abstract}
This work aims to design and develop an optimal vibrotactile seat to provide a high level of satisfaction to the driver. The main critical design parameters were considered and experiments were conducted to investigate the proper values of voltage, frequency, and amplitude that are specifically related to the developed haptic seat.
\end{abstract}

CR Categories: driving simulation, tactile seat, human perception

Keywords: H.5.2 [Information Interfaces and Presentation]: User Interfaces-Haptic I/O, Evaluation/methodology

\section{Introduction}

Driving simulations are widely used to send navigational and warning information to help drivers navigate safely. The visual and auditory channels are highly important for the act of driving; the visual for watching the road and the auditory for listening to warning car horns or other significant sounds. These senses should typically be used in the essential activities of the driving process. Therefore, when the driver resorts to relying on the map of GPS devices or audio directions, he or she undergoes perceptual overload, where too much information is being received through these channels ( $\mathrm{Ji}$ et al. 2011). Haptic-based information in vehicles has major safety implications on reducing visual and auditory overload in driving (Jeon et al. 2013). Given that seats are interfaces that touch the largest area of the driver's body, it is a sensible choice to consider as additional channel of information to the driver.

Many studies focused on vibrotactile feedback in navigation systems for different fields including pilots, car drivers and visually impaired people. Van Erp and et al. (Erp \& Veen n.d.) developed a vibrotactile vest to mentor airplane pilots to the desired destination. They concluded that the employment of the haptic sense to pilots might decrease the high dependency on visual and auditory channels, thereby providing a major safety improvement. For automobiles, the studies can be classified based on the location of vibration: steering wheel, car pedals or driver's seat. Among these studies, Hwang and Ryu (Hwang \& Ryu 2010) employed vibrators in steering wheel to give directional information to drivers. Scientifically, the brain can receive multiple information at the same time, hence using the vehicle seat as a tactile interface is the best option since it has the largest contact to the driver's body (Ji et al. 2011)(Chang et al. 2011).

The aim of this work is to design and develop an optimal vibrotactile seat to provide a high level of satisfaction to the driver.

\footnotetext{
*e-mail: ohalabi@qu.edu.qa

Permission to make digital or hard copies of part or all of this work for personal or classroom use is granted without fee provided that copies are not made or distributed for commercial advantage and that copies bear this notice and the full citation on the first page. Copyrights for third-party components of this work must be honored. For all other uses, contact the Owner/Author.

VRST 2014, November 11 - 13, 2014, Edinburgh, Scotland, UK.

2014 Copyright held by the Owner/Author.

ACM 978-1-4503-3253-8/14/11

http://dx.doi.org/10.1145/2671015.2671134
}

Three main critical design parameters were considered: (1) proper intensity of vibration, (2) position of vibrators and minimum distance between distinguishable vibrations, (3) rhythm of vibration. Experiments were conducted to investigate the proper values of voltage, frequency, and amplitude that are specifically related to the developed haptic seat. A driving simulation was developed for Head-Mounted Display (HMD) to evaluate the haptic interface for the car's seat in a realistic simulation.

\section{System overview}

The system consists of driving set from Logitech ${ }^{\mathrm{TM}}$ to provide natural interface for the driver includes steering wheel, gear shift, and pedals. The tactile seat hardware consists of ten vibrators controlled by Arduino Mega 2560 microcontroller. The controller receives the signal from the software side via wireless serial connection and controls the vibration according to the type of the signal, see Figure 1. The software system is implemented using Vizard software to develop the 3D graphics driving simulation and to control the vibrotactile simulation system by sending the appropriate signal to the tactile seat. A layered approach was considered in development where each layer was responsible for different task like the motions physics of the moving objects, the vehicle state controller to set the navigation and warning tasks and measure the responses, the visual representation which is the $3 \mathrm{D}$ models and the interface with the user.

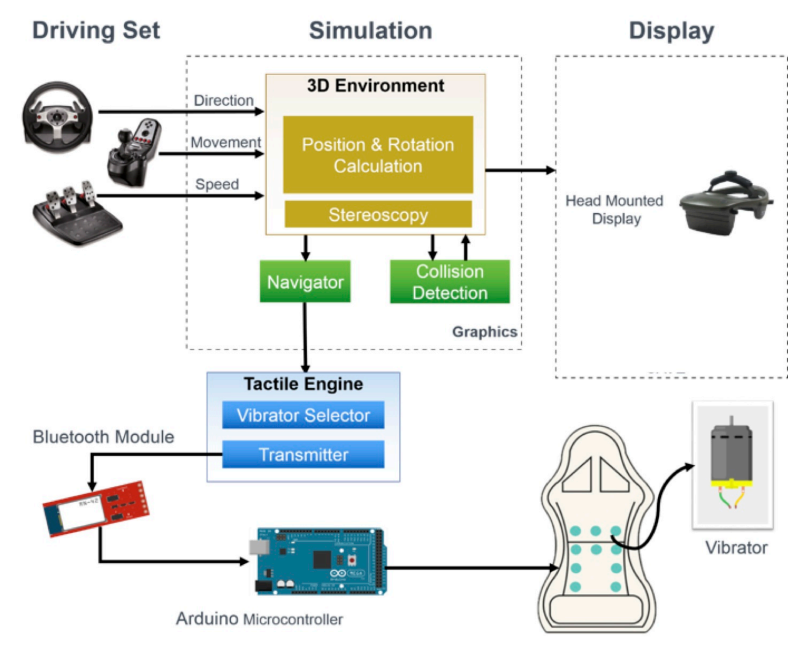

Figure 1. High level system architecture.

\section{Experiments}

Many experiments were conducted using the driving simulation with implemented scenarios where the subject asked to drive according to the navigational signals to get to the goal. One vibrator has been considered in order to find the absolute threshold value of vibration. The threshold considered is when the user noticed or detected the vibration. The absolute threshold was calculated by finding the average point of the transition between 
the threshold when increasing the intensity and threshold when decreasing it based on gender and weight.

\section{Results}

The threshold voltage value for vibration intensity was different between male and female (F: $0.81 \mathrm{~V}, \mathrm{M}: 0.86 \mathrm{~V}$ ), see Figure 2 The weight directly affected the threshold voltage in a proportional manner; the more weight the higher the threshold as in Figure 3.

Regarding the optimum minimum distance between vibrators, the result showed that male and female had almost the same response as can bee seen in Figure 4. The minimum distance vibration sources is $4-6 \mathrm{~cm}$ due to the fact that the perception of two different sources was also affected by the weight. With $4 \mathrm{~cm}$ and weight above $70 \mathrm{Kg}$ the perception was $100 \%$. Never the less, 6 $\mathrm{cm}$ provides $100 \%$ perception for minimum weight of $41 \mathrm{Kg}$. The experiments showed that the participant were more satisfied with the navigational information delivered as vibration compared to audio-based navigation as can bee seen in Figure 5 where $92 \%$ were more satisfied with vibration than audio and only $8 \%$ preferred audio to vibration. The response time for vibration was very close to that of audio and sometime was better but this still need more experiments to and exploration.



Figure 2 The threshold value of vibration intensity in terms of gender.

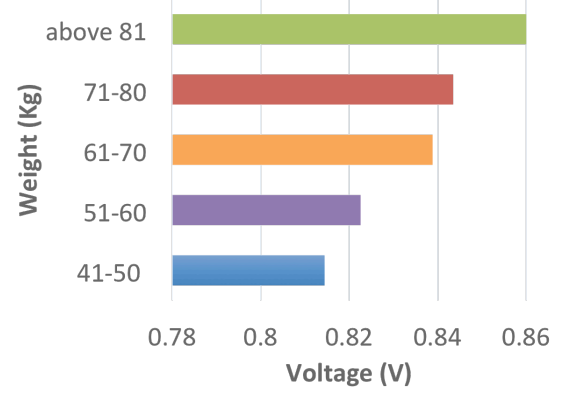

Figure 3 The threshold value of vibration intensity in terms of weight.



Figure 4 Minimum distance between vibration.

\section{Conclusion}

The presented result in this work demonstrates that tactile seat can be considered as an efficient feedback channel to the drivers to reduce the sensory overloading on vision and audio which will lead to safer driving and less traffic accidents. The paper also presented a comprehensive study on how to design the tactile seat and optimize the different design parameters. The threshold voltage value for intensity was slightly less for female than male (F: $0.81 \mathrm{~V}$, M: $0.86 \mathrm{~V})$. The weight directly affected the threshold voltage in linear manner; the more weight the higher the threshold. The results showed that all people (100\%) responded to the navigation in vibration mode correctly, while $75 \%$ responded correctly to the navigation in audio mode. With regard to the response time, the experiments showed that there is no significance difference between audio (5.94 S) and tactile (6.04 S). The overall result of the immersive driving simulation indicated that over $92 \%$ of the people were very satisfied with vibrotactile feedback and preferred it to audio.



Figure 5 Subjects' satisfaction for tactile vs. audio.

\section{Acknowledgment}

This paper was made possible by a UREP award [UREP 14 - 102 - 2 - 035] from the Qatar National Research Fund (a member of The Qatar Foundation). The statements made herein are solely the responsibility of the authors.

\section{References}

Chang, W., Hwang, W. \& Ji, Y.G., 2011. Haptic Seat Interfaces for Driver Information and Warning Systems. International Journal of Human-Computer Interaction, 27(12), pp.11191132.

Erp, J.B.F. Van \& Veen, H.A.H.C. Van, A Multi-purpose Tactile Vest for Astronauts in the International Space Station.

Hwang, S.H.S. \& Ryu, J.R.J., 2010. The Haptic Steering Wheel: Vibro-Tactile Based Navigation for the Driving Environment. Pervasive Computing and Communications Workshops (PERCOM Workshops), 2010 8th IEEE International Conference on, pp.660-665.

Jeon, W. et al., 2013. Assessing the Effectiveness of Vibrotactile Feedback on a 2D Navigation Task., pp.594-600.

Ji, Y.G., Lee, K. \& Hwang, W., 2011. Haptic Perceptions in the Vehicle Seat., 21(3), pp.305-325. 